took place. A fluorescence growth under steady excitation and a phosphorescence decay in the dark were observed at wave-lengths less than those corresponding to the series limit for the respective crystals. This was interpreted in terms of a series of trapping and excited energy-levels in $\mathrm{Tl}^{2+}$ as proposed by Johnson and Williams. A series of experiments on photoconductivity gave negative, though not necessarily conclusive, results at wave-lengths less than that generally accepted for the series limit. Also, an observed sharp rise in fluorescence of KI:Tl at 1000 A. remained unexplained. It was proposed that, in order to explain the latter results, the ionization limits for $\mathrm{KCl}: \mathrm{Tl}$ and $\mathrm{KI}: \mathrm{Tl}$ must be situated at much shorter wave-lengths than the accepted wave-lengths of $1300 \mathrm{~A}$. and $1750 \mathrm{~A}$., respectively.

G. A. Elliotit

\section{COLLOQUE A.M.P.E.R.E., 1957}

T

HE 1957 meeting of the Colloque A.M.P.E.R.E. (Atomes et Molécules par Etudes Radio-Electriques) was held at St. Malo during April 24-26. The conference, which is held annually, is primarily concerned with the study of the properties of materials with the use of radio-frequency electromagnetic waves. The subjects discussed were dielectries, electron paramagnetic resonance, ferromagnetic resonance, microwave spectroscopy of gases, nuclear magnetic resonance, and quadrupole magnetic resonance. The meeting was attended by some two hundred scientists, the majority being from French laboratories, but with visitors from most European countries, including East Germany and Poland and some from as far as Venezuela and Australia. The organizers of the Conference take pride in the fact that the proceedings are conducted almost entirely in French without apparent effort on the part of the foreigners present.

Some seventy papers were presented mainly in the form of ten minute summaries, preprints being available beforehand. This rather heavy programme, combined with splendid weather and French hospitality, made it rather difficult to follow the programme as closely as one might have wished. Prof. R. Freymann, who is the leading spirit of the Colloque A.M.P.E.R.E., was probably tho only person both to attend every session and be at a banquet every evening. Such energy bodes well for French science.

It is evidently impracticable to report each paper and I shall pick out those which I hope will be representative of the Conference. The papers are published in book-form by the Archives des Sciences, and are available from the Institut de Physique, Université de Genève, price 25 Swiss francs. The discussion at the Conference is not recorded or published, as it is feared that this would tend to suppress a free and frank exchange of views.

The session on dielectrics was opened by Prof. $\mathrm{H}$. Fröhlich (Liverpool), who discussed under what conditions trapped electrons at vacancies in ionic crystals would result in dielectric loss of the Debye type. He showed that this may occur, even for single stationary vacancies, when electron lattice interactions are included, whereas previously it has usually been supposed that moving vacancies which simulate a rotating dipole are necessary. S. Dryden (Sydney) reported recent work on Debye-type dielec- tric absorption in ionic crystals, which they have now found in eleven simple ionic crystals and tentatively associate with diffusion of lattice vacancies. M. J. Meinnel and R. Clinet (Rennes) discussed the dielectric effects of the transitions in the ammonium halides, and demonstrated the absolute necessity of removing all traces of water. F. Lasbleis et al. (Rennes) presented results on dielectric absorption and phase changes in a series of titanates. J. Meinnel and F. Trigolet (Rennes) reported dielectric measurements on hexagonal selenium; they find a Debye absorption which is displaced by suitable irradiation, the effect depending on its intensity and wave-length. Prof. R. Freymann (Rennes) reported on various projects, including dielectric measurements on thiourea and on semi-conductors containing various impurities.

Prof. R. H. Cole (Brown University) presented a survey of his dielectric measurements on liquid alkyl halides and mixtures of these with other organic liquids, in the supercooled state. For the mixtures one sometimes finds one dispersion region and sometimes the original two, for which no satisfactory explanation is at present available. J. Ph. Poley and A. J. van Eick (The Hague) presented further results on the two dispersion regions in liquid chloroand bromo-benzenes. L. de Broukère and M. Mandel (Brussels) suggested a method of explaining the spectrum of dielectric relaxation times in high polymers which is based on a consideration of freedom of motion of chain segments. F. C. de Ronde (Eindhoven) proposed a new method of measuring electric and magnetic constants of solids in the microwave region. C. Brot and A. Soulard (Paris) described a special resonant cavity for the frequency range $100-1,000 \mathrm{Mc} / \mathrm{s}$. which appears to facilitate measurements in this difficult region.

In the sessions on electron paramagnetic resonance there were several papers on free radicals. G. Berthet (Fontenay) has studied some stable free radicals, particularly with regard to the hyperfine structure. Some new radicals have been discovered by Y. Fellion and J. Uebersfeld (Paris). F. W. Heineken and F. M. Schimmel (Amsterdam) find that some stable free radicals may be sensitive to oxygen, just like induced ones. M. Bruma (Paris) described a ferrite circulator which seems to have advantages over the 'magic $T$ ' and similar devices used in paramagnetic resonance equipment. D. J. E. Ingram and J. F. Gibson (Southampton) gavo results for radicals formed by ultra-violet irradiation of glassforming substances at low temperatures. D. $\mathrm{H}$. Whiffen et al. (Birmingham) have studied a number of polymers under gamma-irradiation and J. S. van Wieringen (Eindhoven) has investigated glasses subjected to several types of irradiation. At the moment the interpretation of these effects is rather uncertain. D. J. E. Ingram (Southampton) described how structural data on large molecules may be obtained from electron resonance in favourable cases, for example, with hæmoglobin. J. Uebersfeld and E. Erb (Paris) reported further work on paramagnetic centres in carbon. E. E. Schneider (Newcastle) reported further results on alkali halides doped with manganese and on $\mathrm{X}$-irradiated lithium fluoride. A. Gozzini et al. (Pisa) have measured magnetic birefringence effects using DPPH.

The ferromagnetic resonance in a single crystal of iron has been studied by A. Strub (Grenoble). J. Munier and P. Sermet (Grenoble) have developed a ferrite microwave attenuator with 'electronic' control. 
The session on nuclear magnetic resonance was introduced by Prof. C. J. Gorter (Leyden), who reported on current work in this field in his laboratory. N. J. Poulis and G. E. G. Hardemann (Leyden) reported further measurements on a single crystal of cupric chloride. A. Lösche (Leipzig) reported on the use of nuclear resonance in studying the rate of slow polymerization by comparing the signal from protons in the solid (polymer) and liquid (monomer) phases. R. Extermann (Geneva) gave a review of the work of the Geneva group which has been particularly concerned with magnetic resonance at low fields. A. Abragam and W. Proctor (Saclay) discussed their work on 'spin calorimetry', one result of which is a trick for polarizing nuclei which have an inconveniently long spin-lattice relaxation time (weeks, say) in a matter of seconds. A. Abragam and I. Solomon (Saclay) have studied the spin echoes obtained when a nuclear absorption line is subject to quadrupole splitting. The results differ considerably from the usual ones, in that 'echoes' may be observed at $\tau / 2$ and $3 \tau / 2$ as well as $\tau$ after the stimulation. Abragam's group have also observed the Overhauser effect in liquids containing paramagnetic impurities which has the peculiar property that there is at first a dim. inution in the nuclear signal (Underhauser effect). The increase in intensity obtainable could be invaluable in the study of weakly magnetic nuclei. J. G. Powles (London) discussed measurements on polyisobutylene solutions in organic liquids over a wide range of concentration which give information on chain motion in this polymer. J. Hue and J. Seiden (Paris) have produced a simplified theory of double resonance.

A study of hyperconjugation in molecules by means of microwave spectroscopy was reported by J. Sheridan et al. (Birmingham). Results by the same method on formic acid were given by $\mathrm{R}$. Wertheimer (Paris). J. Bonanomi et al. (Neuchâtel) have constructed an ammonia 'maser' and have studied particularly the reproducibility of such equipment. This communication provoked the usual argument about the meaning of time. Prof. A. Kastler (Paris) gave a review of his work on magnetic resonance in optically excited systems of various types.

The last session was concerned with nuclear quadrupole resonance. Prof. Kastler pointed out that Maupertuis, a native of St. Malo, was the first to measure the departure of the earth from sphericity and hence was one of the first to measure a quadrupole moment. F. Lurcat (Paris) showed how one can obtain macroscopic equations of motion for quadrupole resonance absorption corresponding to the Bloch equations for nuclear resonance. M. Buyle-Bodin (Grenoble) has studied the phase transformations in $p$-dichlorobenzene by means of quadrupole resonance.

The Conference was organized by Prof. R. Freymann (Rennes) and Dr. J. Le Bot (St. Malo-Rennes), and was supported financially by French industry. The conference was made royally welcome by the Municipality of St. Malo. The publication of the report and preparation of preprints were undertaken again by Dr. G. J. Béné and the Geneva group.

The Colloque A.M.P.E.R.E. 1958 will be held in Paris during June. Information concerning the Colloque A.M.P.E.R.E. can be obtained from Prof. R. Freymann, University of Rennes, or from Dr. J. G. Powles, Physics Department, Queen Mary College, Jondon, E.I.
J. G. POWLES

\section{THE THIRD LAW OF THERMODYNAMICS}

$T$ THE "Year Book of the Physical Society, 1956", the second in the series, contains the texts of the fortieth Guthrie Lecture and the eleventh Holweck Lecture; the addresses delivered by the recipients of the thirty-second Duddell Medal and the eleventh Charles Vernon Boys Prize; abstracts of the dis. courses given at the fortieth Physical Society exhibition of scientific instruments and apparatus ; obituary notices of fourteen fellows including Irene JoliotCurie, A. O. Rankine, H. R. Robinson, S. Whitehead and R. W. Wood; and the report of the Council of the Society for 1955 .

In his Guthrie Lecture which he delivered on March 13, 1956, the late Sir Francis Simon gives a historical and eritical survey of the third law of thermodynamics. In 1906, in a paper entitled "On the Calculation of Chemical Equilibria from Thermal Measurements", W. Nernst enunciated his so-called Heat Theorem which has now become the third law, and although at first it was considered mainly as a means of predicting chemical equilibria and directly applicable only to condensed systems, its later formulation as the law of the unattainability of absolute zero, and its extension to gaseous systems, led to Nernst's claim that it was a law of general validity. However, subsequent investigations disclosed a number of notable discrepancies, and the law was rejected by many physicists. In 1927 , Simon gave a new formulation of the law, which states that the entropy of all factors within a system that are in internal thermodynamic equilibrium disappears at absolute zero, and in this form the law covers all cases without exception. Sir Francis mentions that he was first a pupil, then a research assistant and finally a colleague of Nernst, and was thus close enough to obtain a very clear picture of what Nernst had in mind, but yet not so close as to be completely dominated by Nernst. In fact, some of the strongest opposition to Simon's new formulation of the third law came from Nernst himself. Turning to recent experimental developments, Sir Francis deals with spin systems, and indicates the various sub-systems which must exist within a substance, some of which are in internal equilibrium and lose their entropy at some low temperature, and others in which the disorder persists down to the lowest temperatures. Finally, he poses the question as to the present usefulness of the third law, and maintains that it has outlived most of its usefulness in the field for which it was originally intended, namely, chemical gas reactions, since the corresponding condensed phases may not be in internal thermodynamic equilibrium. Absolute zero of temperature is no longer a point of reference for the thermodynamic calculations in these cases, and we must take the quantum statistical expression for the entropy of the gas to find out whether the condensed phase at absolute zero is in perfect order or whether it has a frozen-in disorder. In low-temperature physics, however, the third law is a most important guide since most of the systems of interest to physicists are not liable to become frozen in. Moreover, those cases where apparent discrepancies with the third law have been discovered have led to a better understanding of many properties of the condensed phase.

The Holweck Lecture, entitled "The Diffusion of Visible Light by Cubic Crystals", was delivered by 